\title{
Analytical dynamic and quasi-static model of railway vehicle transit to curved track
}

\author{
HABIB BetTAIEB ${ }^{\mathrm{a}}$ \\ École préparatoire aux académies militaires de Sousse, Tunisie \\ Laboratoire de génie mécanique, École nationale d'ingénieurs de Monastir, avenue Ibn-Eljazzar, 5000 Monastir, Tunisia
}

Received 12 December 2011, Accepted 27 June 2012

\begin{abstract}
This paper shows that the dynamic study of a railway vehicle in curve can be reduced to the study of a bogie and two wheelsets in the quasi-static case. Every wheelset supports the load of the quarter of the vehicle. From this model, we can optimize the suspension to define the mechanical build characteristics of vehicle.
\end{abstract}

Key words: Simulation / curving behaviour / dynamic and quasi-static model of railway vehicle / Circulation in tight curve / lateral -creep force / longitudinal creep force

Résumé - Modèle analytique dynamique et quasi-statique d'un véhicule ferroviaire en circulation sur une voie en courbe. Cet article montre que l'étude dynamique d'un véhicule ferroviaire en circulation sur une voie en courbe, peut être réduite à l'étude d'un bogie à deux essieux dans le cas quasi-statique. Chaque essieu supporte la charge du quart du véhicule. De ce modèle, on peut optimiser la suspension afin de définir les caractéristiques mécaniques du véhicule d'une façon précise.

Mots clés : Simulation / comportement en courbe / modèle dynamique / modèle quasi-statistique / circulation en courbe de faible rayon / force de pseudo-glissement latérale / force de pseudo-glissement longitudinale

\section{Introduction}

The research for a long life span of the railway vehicles and for safety obliged railway companies to plan big investments to build ways of high geometrical, rectilinear qualities and curves of big radius. The majority of the existing vehicles are optimized in stability in alignment for high speed, but not for the traffic of short radius curve. Such a matter induces problems of maintenance of both wheel and rail. Among these problems, we notice that the wear of the wheel as well as that of the rail cost much money to railway companies and create the risk of derailment. A number of investigations have worked on improving the rail vehicle design between the stability and the curving performance.

Bettaieb [1] developed also a model using a rail inclination. This model studies the variation of rail inclination on the motion of the vehicle. This will allow us to master the transverse displacement of the wheelset, to avoid flange contact in a curve, which causes high wear on the wheel and the rail while preserving a good stability in alignment. This will reduce the cost of maintenance

\footnotetext{
a Corresponding author:

Habib. bettaieb@enim.rnu.tn
}

and will improve the comfort of the passengers. Bettaieb et al. [2] used the model of a complete vehicle for the study of the vehicle stability at high speed and the quasi-static model of bogie and two wheelsets for the study of the traffic in short radius curved tracks. They used the genetic algorithms for simple objective and multi-optimization. Indeed, they showed that this method always allows for finding the optimal values of several variables (parameters of construction of the vehicle). These values allow the vehicle to traffic with a very high speed with safety in alignment. These same optimal values allow the vehicle to traffic without sliding, and without flange contact between the wheel and the rail for the big and short radius curved tracks. Bettaieb et al. [3,4] used the model to study the quasi-static model of bogie and two wheelsets for the study of the traffic in short radius curved tracks. Indeed, they showed that the model is used to optimize the mechanical characteristic vehicles design and analyses of its performance. Boocok et al. [5] considered the steady-state motion of bogie and two-wheelset vehicles. Special attention was given to the effect of primary and secondary suspension parameters and the results were evaluated with experiments. Dukkipati et al. [6] considered the steady state motion of conventional bogie and 


\section{Nomenclature}

\begin{tabular}{|c|c|c|c|}
\hline $2 a$ & Wheelbase & $R_{c}$ & Curve radius \\
\hline $2 \bar{A}$ & $\begin{array}{l}\text { Distance between the } \\
\text { bogies centres }\end{array}$ & $r_{j}$ & $\begin{array}{l}\text { Rolling circle radius of the wheel } \\
\text { at the points of contact }\end{array}$ \\
\hline$C_{11}, C_{22}, C_{23}$ and $C_{33}$ & $\begin{array}{l}\text { Creep coefficients of } \\
\text { Kalker }\end{array}$ & $T_{k i j}$ & Lateral creep forces \\
\hline$e_{j}$ & $\begin{array}{l}\text { Distance between the centre } \\
\text { of inertia of the wheelset and } \\
\text { the wheel and rail points of } \\
\text { contact }\end{array}$ & $V$ & Vehicle velocity \\
\hline$e_{0}$ & $\begin{array}{l}\text { Half distance between the } \\
\text { contact points in the } \\
\text { central position }\end{array}$ & $X_{k i j}$ & Longitudinal creep forces \\
\hline$K_{x}$ & $\begin{array}{l}\text { Longitudinal stiffness of } \\
\text { primary suspension }\end{array}$ & $Z$ & Height of wheelset centre \\
\hline$\overline{K x}$ & $\begin{array}{l}\text { Longitudinal stiffness of } \\
\text { secondary suspension }\end{array}$ & $\gamma_{e}$ & Wheel equivalent conicity \\
\hline \multirow[t]{2}{*}{$\overline{K y}$} & $\begin{array}{l}\text { Lateral stiffness of } \\
\text { secondary suspension }\end{array}$ & & \\
\hline & & $\begin{array}{l}\gamma_{n c} \\
\gamma_{0}\end{array}$ & $\begin{array}{l}\text { Not compensated acceleration } \\
\text { Wheel conicity (new wheel) }\end{array}$ \\
\hline$M_{k i j}$ & Spin creep moment & $\nu_{k i j}$ & Creepages at the contact point \\
\hline$m$ & Wheelset mass & $\left\{\begin{array}{l}\rho_{x k i} \\
\rho_{y k i} \\
\rho_{z k i}\end{array}\right.$ & $\begin{array}{l}\text { Radius of inertia of the } \\
\text { wheelset about axis } \\
\left(G_{0 k i} \vec{x}_{\delta p k i}, G_{0 k i} \vec{y}_{\delta p k i}, G_{0 k i} \vec{z}_{\delta p k i}\right) \\
\text { respectively }\end{array}$ \\
\hline$\underline{M}$ & Bogie mass & $\mu$ & Friction coefficient \\
\hline $\bar{M}$ & Body mass & $\omega_{N k i j}$ & $\begin{array}{l}\text { Angular velocity about normal in } \\
\text { Contact point } j\end{array}$ \\
\hline$N$ & Normal load for a wheel & $\left(\frac{\overline{\Omega_{x}}}{\frac{\Omega_{y}}{\Omega_{z}}}\right.$ & $\begin{array}{l}\text { Radius of inertia of the } \\
\text { body about axis } \\
\left(\bar{G} \vec{x}_{\delta p}, \bar{G} \vec{y}_{\delta p}, \bar{G} \vec{z}_{\delta p}\right) \text { respectively }\end{array}$ \\
\hline$R^{\prime}$ & Lateral radius of rail profile & $\begin{array}{l}\Omega_{x k} \\
\Omega_{y k} \\
\Omega_{z k}\end{array}$ & $\begin{array}{l}\text { Radius of inertia of } \\
\text { the bogie about axis } \\
\left(O_{0 k} \vec{x}_{\delta p k}, O_{0 k} \vec{y}_{\delta p k}, O_{0 k} \vec{z}_{\delta p k}\right) \\
\text { respectively }\end{array}$ \\
\hline$R$ & $\begin{array}{l}\text { Transverse radius of wheel } \\
\text { profile }\end{array}$ & $\sigma \mathrm{k}$ & $\begin{array}{l}\text { Angles between the longitudinal } \\
\text { axis of the body and bogies. }\end{array}$ \\
\hline$r_{0}$ & $\begin{array}{l}\text { Rolling circle radius for } \\
\text { a central position }\end{array}$ & $\sigma \mathrm{ki}$ & $\begin{array}{l}\text { Angles between the longitudinal axes of } \\
\text { the bogies and middle of the wheelset. }\end{array}$ \\
\hline$\delta$ pki & $\begin{array}{l}\text { Superelevation to the } \\
\text { wheelset position }\end{array}$ & $\delta \mathrm{pk}$ & $\begin{array}{l}\text { Superelevation of the bogies } \\
\text { position }\end{array}$ \\
\hline$\delta \mathrm{p}$ & $\begin{array}{l}\text { Superelevation of the } \\
\text { body position }\end{array}$ & & \\
\hline
\end{tabular}

two-wheelset vehicle, the effects of unsymmetrical suspensions were also included. Many designs are considered to provide good dynamic performance and the ability of the vehicle to steer around a curve. Bettaieb [10] has developed a thesis, which leads to the conclusion that in the design of the railway vehicle there is a conflict between dynamic stability at a high speed and the ability of the vehicle to steer around curves.

To approach most real systems, it is necessary to adopt a mathematical model, which describes well the system to be studied. The aim of this work, is to find the mathematical model, which describes the behaviour of complete vehicle in curve.

\section{Vehicle system model}

We define with precision the parameters used in the mechanical model. A railway vehicle is taken as a multiple rigid body system composed of wheelsets, bogie frames, and car body (see Fig. 1). These rigid bodies are connecting to each other by elements of suspension, primary $K x ; K y ; K z$ and secondary suspension $\overline{K x} ; \overline{K y} ; \overline{K z}$. The 


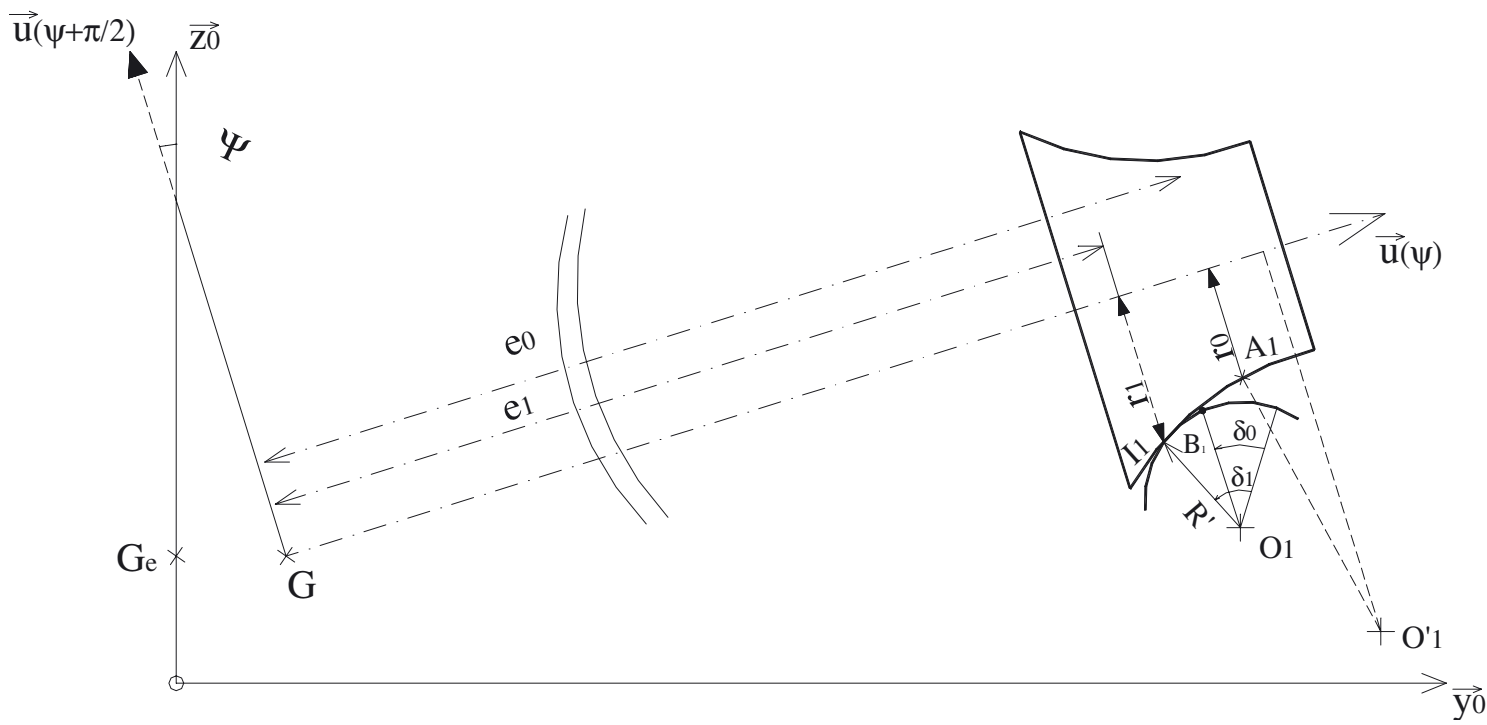

Fig. 1. Wheelset parameters.

Table 1. Degrees of fredoom of the vehicle.

\begin{tabular}{cccc}
\hline & lateral displacement & yaw & roll \\
\hline Bogie $\left(C_{k}\right): k=1,2$ & $y_{k}$ & $\alpha_{k}$ & $\theta_{k}$ \\
Car body $(\mathrm{C})$ & $\bar{Y}$ & $\bar{\alpha}$ & $\bar{\theta}$ \\
Wheelsets $\left(S_{k i}\right) \quad k, i=1,2$ & $y_{k i}$ & $\alpha_{k i}$ & $\psi_{k i}=\Gamma \cdot y_{k i}$ \\
\hline
\end{tabular}

dampers are putting in parallel with the springs. The steps followed in this process begin with the geometrical study of the wheel and rail contact. This study takes linear wheel/rail geometry with two points of contact into account. The distances $e_{j}, r_{j}$ between the centre of inertia of the wheelset and the wheel and rail points of contact are a function of the position parameters of the wheelset [10] as shown in Figure 3. These expressions are given by Eqs. (A.1)-(A.6). The application of the Kalker's theory [9] determines the actions of contact between the rail and the wheel. It should be mentioned that for the investigations of the curving behaviour, the values of the body's centrifugal forces due to lateral curvature of the truck's central line are introduced. It is assumed that the lateral and the vertical vibrations of the system are uncoupled, thus, only the lateral vibrations of the system are taken into consideration for this analysis. The wheelset is given with an index $i=1$ (leading wheelset) or $i=2$ (trailing wheelset), a contact point on the wheel tread is indicated by the index $j=1$ (left wheel in the direction of travel) and $j=2$ (right wheel). The bogie frame is given with an index $k=1$ (the first in the direction of travel), and $k=2$ (the second). For all solids, the movements considered are the lateral displacement, the yaw and the roll. These movements are noted in Table 1.

\subsection{Used references and parameters}

We must clearly define the notations and reference frame used for the passage from one basis to another.
The parameter setting of different solids in the vehicle moving from the alignment to the connecting curve is as follows:

$\overline{O_{0}}$ : mobile centre related to the car body,

$O_{O k}$ : mobile centre related to the bogie $C_{k}$,

$O_{O k i}$ : mobile centre related to the wheelsets $S_{k i}$.

$O_{0 k i}$ : moving along the axis track geometry.

The plans $\left(O_{g}, \vec{x}_{g}, \vec{y}_{g}\right)$ and $\left(\overline{O_{0}}, \vec{x}_{0}, \vec{y}_{0}\right)$ are parallel. The basis of $R_{0}$ is deduced from the basis of $R g$ by a rotation $\sigma$ (axis $\vec{z}_{0}=\vec{z}_{g}$ ). The basis of $R_{0 k}$ is deduced from the basis of $R_{0}$ by a rotation $\sigma_{k}$ (axis $\vec{z}_{0 k}$ ). The basis of $F_{k i}$ is deduced from the basis $R_{0 K}$ by a rotation $\sigma_{k i}$ (axis $\vec{b}_{k i}$ ). The basis of $R_{\delta p k i}$ is deduced from the basis of $F_{k i}$ by a rotation $\sigma_{k i}$ (axis $\vec{z}_{\delta p k i}$ ). The basis of $R_{\delta p}=\left(\overline{O_{0}}, \vec{x}_{\delta p}, \vec{y}_{\delta p} ; \vec{z}_{\delta p}\right)$ is deduced from the basis of $R_{0}$ by a rotation $\delta p$ (axis $\vec{x}_{0}=\vec{x}_{\delta p}$ ). The basis of $R_{\delta p k}=$ $\left(O_{0 k}, \vec{x}_{\delta p k}, \vec{y}_{\delta p k}, \vec{z}_{\delta p k}\right)$ is deduced from the basis $\left(R_{0 k}\right)$ by a rotation $\delta p k$ (axis $\left.\vec{x}_{0 k}=\vec{x}_{\delta p k}\right)$. The basis of $R_{\delta p k i}=$ $\left(O_{0 k i}, \vec{x}_{\delta p k i}, \vec{y}_{\delta p k i}, \vec{z}_{\delta p k i}\right)$ is deduced from the basis of $F_{k i}$ by a rotation $\delta p_{k i}\left(\right.$ axis $\vec{x}_{\delta p k i}=\vec{b}_{k i}$ ) (see Fig. 2 ).

\subsection{Expressions of force and moment of centrifugal and Coriolis force}

The expressions of force and moment of centrifugal for the car body, bogie, wheelset; of force and moment inertia Coriolis wheelset's are given by Appendix A Eqs. (A.7)-(A.10). 


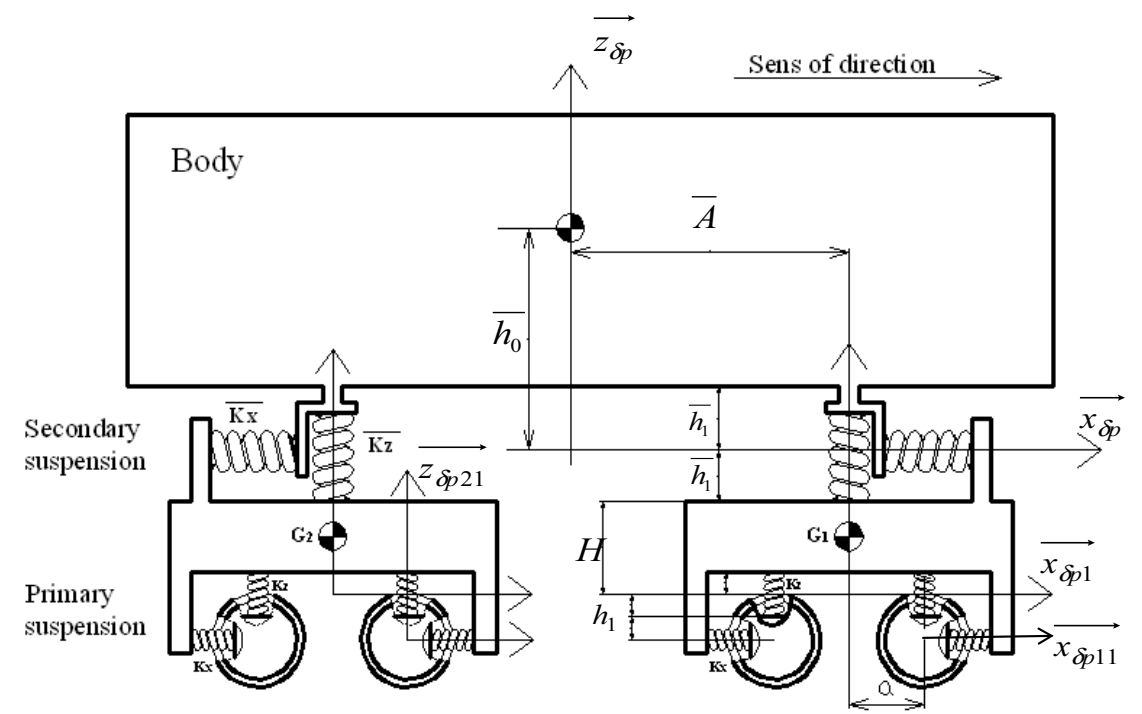

Fig. 2. Configuration of a 17 DOF rail vehicle model.

Table 2. Used references.

\begin{tabular}{cc}
\hline$R_{g}=\left(O_{g}, \vec{x}_{g}, \vec{y}_{g}, \vec{z}_{g}\right)$ & Galilean reference attached to the curve centre. \\
$R_{0}=\left(\vec{O}_{0}, \vec{x}_{0}, \vec{y}_{0}, \vec{z}_{0}\right)$ & moving reference attached to the car body. \\
$R_{0 k}=\left(O_{0 k}, \vec{x}_{0 k}, \vec{y}_{0 k}, \vec{z}_{0 k}\right)$ & moving reference fixed to the bogie $C_{k}$. \\
$F_{k i}=\left(O_{0 k i}, \vec{t}_{k i}, \vec{n}_{k i}, \vec{b}_{k i}\right)$ & Frenet frame. \\
\hline
\end{tabular}

\subsection{Kinetic energy of the vehicle}

Having completely defined the coordinate system, we calculate the kinetic energy (see Appendix A (Eq. (A.11)).

\subsection{Power developed by the springs}

Equation (A.12) gives the power developed by the springs and dampers.

\subsection{Power developed by the action of gravity}

The power developed by the gravity is given by Appendix A Eq. (A.13).

\subsection{Wheel and rail interactive forces}

The traffic of vehicle in a tight curve $(R c<500 \mathrm{~m})$, the reduced sliding of wheels become more important. In that case, the force and moment contact are obtained by applying the law of saturation proposed by Johnson and Vermeulen [8], based on the coefficients of Kalker. The calculus of the wheel and rail interactive forces is given by Appendix A (Eqs. (A.14)-(A.18)).

\subsection{Dynamic model}

The set of differential equations is obtained by applying Lagrange's equations, with $q_{i}$ : generalized coordinate vector, $q_{i}^{\prime}$ : derived from the generalized coordinate vector, $T^{g}(\mathrm{D})$ : kinetic energy, $\Pi_{i}^{g}\left\{\overline{S_{j}} \rightarrow S_{j}\right\}$ : coefficient of $q_{i}$ ' in expression of power.

$$
\begin{aligned}
\frac{\mathrm{d}}{\mathrm{d} t} \frac{\partial T^{(\lambda)}(D)}{\partial q_{i}^{\prime}}-\frac{\partial T^{(\lambda)}(D)}{\partial q_{i}} & = \\
\sum_{j=1}^{j=n} \Pi_{i}^{(\lambda)}\left\{\overline{S_{j}}\right. & \left.\rightarrow S_{j}\right\}-\sum_{j=1}^{j=n}\left\{\begin{array}{l}
\lambda \\
S_{j}, q_{i}
\end{array}\right\} \\
& \times\left\{\left\{\left.A_{S_{j}}^{e}\right|_{\lambda} ^{g}\right\}+\left\{A_{S_{j}}^{c}||_{\lambda}^{g}\right\}\right\}
\end{aligned}
$$

$\left\{\left.A_{S_{j}}^{e}\right|_{\lambda} ^{g}\right\}$ corresponds to inertia centrifugal force and moment of $(\mathrm{Sj})$ in movement of $\lambda$ relative to the fixed reference (Rg); $\left\{\left.A_{S_{j}}^{c}\right|_{\lambda} ^{g}\right\}$ to Coriolis inertia force and moment of solid $(\mathrm{Sj})$ in movement of $\lambda$ relative to the fixed $(\mathrm{Rg})$ and $\left\{\begin{array}{l}\lambda \\ S_{j}, q_{i}\end{array}\right\}=\frac{\partial}{\partial q_{i}^{\prime}}\left\{\begin{array}{l}\lambda \\ S_{j}\end{array}\right\} ;\left\{\begin{array}{l}\lambda \\ S_{j}\end{array}\right\}$ define the rotation and speed of solid $S_{j}$. The use of Lagrange's equation gives us the dynamic equations. These equations stimulate the dynamic behaviour of a vehicle in traffic of transition curve of radius $R c$. The curves of transition have an important role in the quality of the comfort, particularly for the high-speed vehicles. The dynamic model developed in this research can simulate the dynamic vehicle of transition curve. 


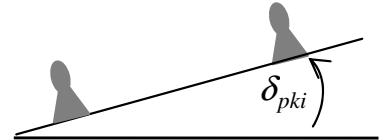

\subsection{Motion on a curve}

Some hypotheses were adopted for the dynamic study of the vehicle in traffic on a way in curve: the vehicle travels with a constant speed; the system is at equilibrium; the curve radius $R_{C}$ is constant. Then we can neglect the damping forces and the inertia forces compared to the elastic forces. The rolls of the car body and bogie in the motion on a curve are small and we can write:

$$
\begin{gathered}
\bar{\theta}=\theta_{k}=0 ; \delta_{p k i}=\delta_{p k}=\delta_{p}=0.07 \mathrm{rd} ; \\
\gamma_{n c}=\frac{V^{2}}{R c}-g . \delta p-g . \delta p \text { and } W=\left(m+\widehat{m}+\frac{M}{2}+\frac{\bar{M}}{4}\right) \cdot g \\
\rho_{11}=-\rho_{12}=-\rho_{21}=-\rho_{22}=\frac{1}{R c} ; \sigma_{1}=-\sigma_{2}=\frac{-\bar{A}}{R c} \\
\sigma_{11}=-\sigma_{12}=\sigma_{21}=-\sigma_{22}=\frac{-a}{R c}
\end{gathered}
$$

\subsubsection{Variable of relative positions}

We propose that the vehicle is rigid with nominal geometry describing perfect track design. The track centre line, which is an element of nominal geometry, always stays in horizontal plan. In a curve of constant radius, a free motion wheelset in a railway takes a stable position, which corresponds to a motion of rolling without slip. This position has a lateral displacement [10]:

$$
y_{0}=\frac{e_{0} \cdot r_{0}}{\gamma_{e} \cdot R_{c}}
$$

We take this position as the origin of the lateral displacement, and we write:

$$
\begin{aligned}
\bar{Y}= & \frac{\overline{Y_{1}}+\overline{Y_{2}}}{2} ; \bar{\alpha}=\frac{\overline{Y_{1}}-\overline{Y_{2}}}{2 \bar{A}} ; \overline{Y_{1}}=\overline{Y_{1}^{*}}+Y_{1}^{*}+\frac{y_{11}^{*}+y_{12}^{*}}{2}+y_{0} ; \\
\alpha_{k}= & \alpha_{k}^{*}+\frac{y_{k 1}^{*}-y_{k 2}^{*}}{2 a} ; \overline{Y_{2}}=\overline{Y_{2}^{*}}+Y_{2}^{*}+\frac{y_{21}^{*}+y_{22}^{*}}{2}+y_{0} ; \\
& y_{k}=y_{k}^{*}+\frac{y_{k 1}^{*}+y_{k 2}^{*}}{2}+y_{0} ; y_{k i}=y_{k i}^{*}+y_{0} ; \alpha_{k i}
\end{aligned}
$$

$\overline{Y_{1}}$ and $\overline{Y_{2}}$ are the lateral displacements of two points of the car body. These points belong to the axis of the elastic connection between bogie and the car body. They coincide with the geometrical axis of two bogies in position at rest. The dynamic equations of the vehicle in the quasi-static case is: $\mathrm{M}(14,14)(\mathrm{X}) * \mathrm{X}=\mathrm{B}$ (Eq. (A.19)).

\subsubsection{Decomposition of the rigidity matrix}

The value of the longitudinal rigidity of the secondary suspension $\overline{K x}$ is low in regard to $K x$ and $K y$ values, this condition makes the term $\overline{K x} \cdot \overline{d^{2}} / \bar{A}$ be neglected, because $2 \bar{A}$, the distance between the bogies centres, is high. From Equation (A.19), the relations relative to the car body are:

$$
\left\{\begin{array}{l}
2 \overline{K_{Y}} \cdot \bar{A} \cdot \overline{Y_{1}^{*}}-2 \overline{K_{Y}} \cdot \bar{A} \cdot \overline{Y_{2}^{*}}=0 \\
2 \overline{K_{Y}} \cdot \overline{Y_{1}^{*}}+2 \overline{K_{Y}} \cdot \overline{Y_{2}^{*}}=\bar{M} \gamma_{n c}
\end{array} \quad \Rightarrow \overline{Y_{1}^{*}}=\overline{Y_{2}^{*}}=\frac{\bar{M} \cdot \gamma_{n c}}{4 \overline{K_{Y}}}\right.
$$




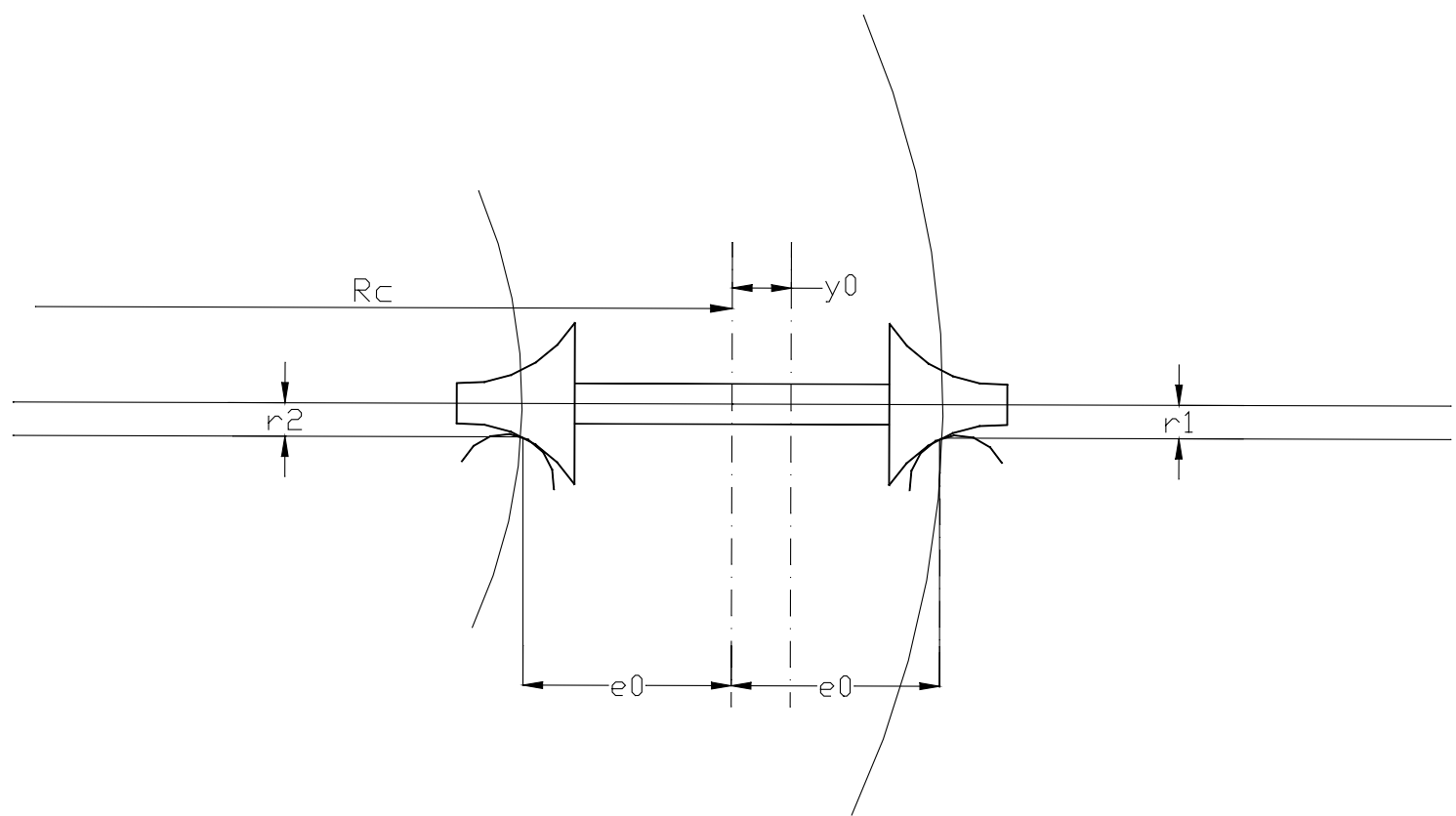

Fig. 4. Wheelset position in a curve.

We replace the expressions of $\overline{Y_{1}^{*}} \& \overline{Y_{2}^{*}}$ in the relations relative of bogie Equation (6) and we obtain:

$$
\begin{aligned}
& \left\{\begin{array}{l}
4 K_{Y} \cdot Y_{1}^{*}-.2 \overline{K_{Y}} \cdot \overline{Y_{1}^{*}}=M \gamma_{n c} \\
4 K_{Y} \cdot Y_{2}^{*}-.2 \overline{K_{Y}} \cdot \overline{Y_{2}^{*}}=M \gamma_{n c}
\end{array}\right. \\
& \Rightarrow Y_{1}^{*}=Y_{2}^{*}=\frac{\left(M+\frac{\bar{M}}{2}\right) \cdot \gamma_{n c}}{4 K_{Y}}
\end{aligned}
$$

We find the expressions above of the lateral displacement of the car body and the two bogies. The decoupling of the dynamic equations Equation (A.19) gives two uncoupled systems Equation (A.20) which describe the motions of the two identical bogies and the four wheelsets.

$$
\begin{aligned}
& {\left[\begin{array}{ll}
{[M(5,5)]} & 0 \\
0 & {[M(5,5)]}
\end{array}\right] }\left\{\begin{array}{l}
X_{1} \\
X_{2}
\end{array}\right\}=\left\{\begin{array}{l}
B \\
B
\end{array}\right\} ; \\
& {[M(5,5)]\left(X_{1}\right) .\left\{X_{1}\right\}=\{B\} }
\end{aligned}
$$

Each bogie and two wheelsets have 5 degrees of freedom $\left\{X_{1}\right\}=\left\{\alpha_{1}^{*}, y_{1 i}^{*}, \alpha_{1 i}\right\}^{T}$ and $\left\{X_{2}\right\}=\left\{\alpha_{2}^{*}, y_{2 i}^{*}, \alpha_{2 i}\right\}^{T} i=$ 1,2 . We have showed that a bogie and two wheelsets Equation (A.20) can replace the semi-static behaviour of the complete vehicle in curve.

We define a vectorial sub-space $\left(Y_{1}^{*} ; \alpha_{1}^{*} ; y_{11}^{*}, \alpha_{11}\right.$; $\left.y_{12}^{*} ; \alpha_{12}\right)$ of the decoupled bogie and two wheelsets, the additional sub-space $\left(\overline{Y_{1}^{*}} ; \overline{Y_{2}^{*}} ; Y_{2}^{*} ; \alpha_{2}^{*} ; y_{21}^{*}, \alpha_{21} ; y_{22}^{*} ; \alpha_{22}\right)$ of the complete vehicle. We note $\operatorname{MS}(8,8)(\mathrm{X})$ the additional matrix (Eq. (A.21)).

\subsubsection{Derailment force}

For the traffic on a way in curve, we suppose the vehicle travels with a constant speed and the curve radius

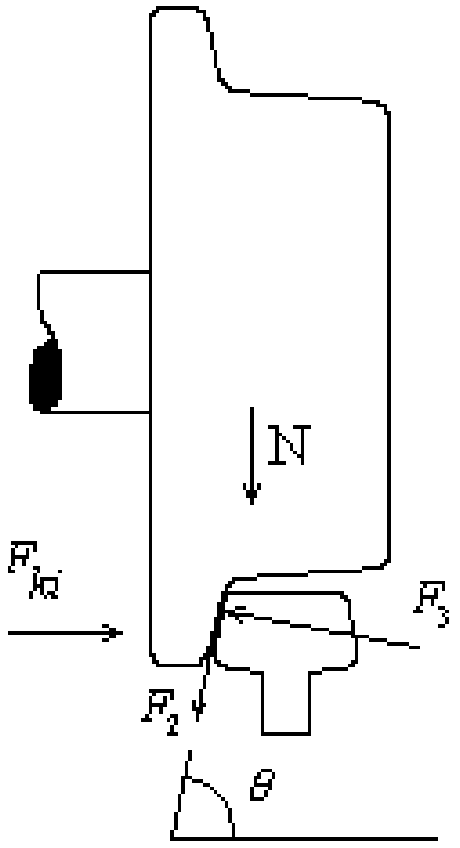

Fig. 5. Lateral effort $F_{k i}$.

is constant. The play between rail and wheel is $0,01 \mathrm{~m}$. If the wheelset lateral displacement $y_{k i}=y_{k i}^{*}+y_{0}$ is equal at the play between rail-wheel, the variable $y_{k i}$ is constant and presents known value. At this moment, the wheelset touches the rail and exercises a lateral effort $F_{k i}[2]$. To avoid the derailment [7], it is necessary that:

$$
\frac{F_{k i}}{N}<\frac{t g \theta_{\max }-\mu}{1+\mu \cdot \operatorname{tg} \theta_{\max }}
$$

We supervise the value of the lateral displacement $y_{k i}$. If the lateral displacement $y_{k i}$ is equal or greater than 


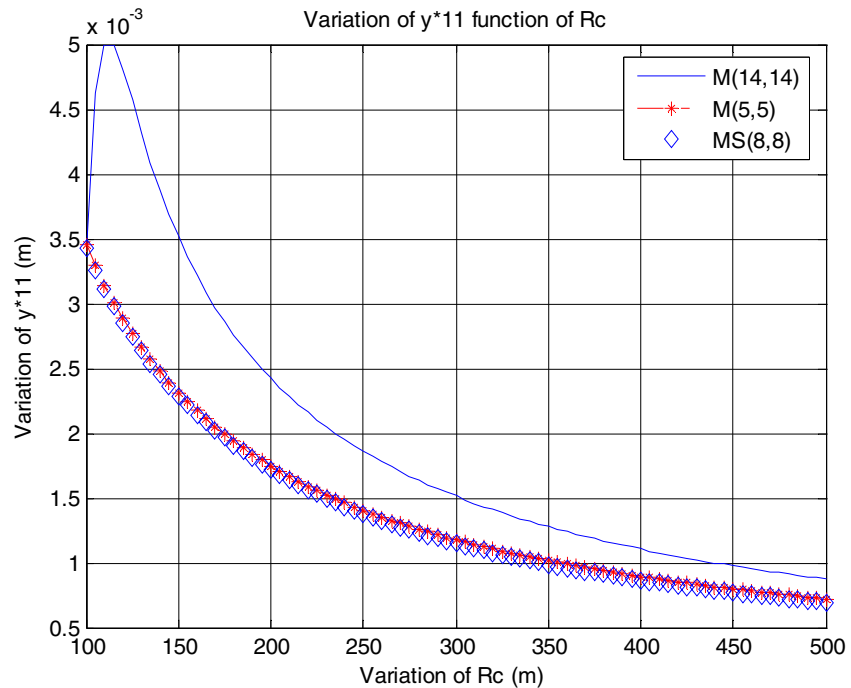

Fig. 6. Leading wheelset lateral displacement $y_{11}^{*}$ as function of $R c$.

play $=10 \mathrm{~mm}$, there is an effort $F k i$, otherwise $F k i=0$. The calculation of the lateral forces $F_{1 i}$ applied by each wheelset $(i=1,2)$ on the rail is obtained from:

- Line (1 or 3) of matrix M(14, 14)(X) Equation (A.19);

$$
\begin{array}{r}
F_{1 i}=(m+\widehat{m}) \cdot \gamma_{n c}-W \cdot \phi \cdot\left(y_{0}+y_{1 i}^{*}\right)+2 \cdot \chi \cdot C_{22} \cdot V J X_{1 i} \cdot \alpha_{1 i} \\
+2 \cdot K y \cdot Y_{1}^{*}+(-1)^{i+1} \cdot 2 \cdot K y \cdot a \cdot \alpha_{1}^{*}
\end{array}
$$

- Line (1 or 3) of matrix $\mathrm{M}(5,5)\left(\mathrm{X}_{1}\right)$ (Eq. (A.20)).

$$
\begin{array}{r}
F_{1 i}=W \cdot \gamma_{n c}-W \cdot \phi\left(y_{0}+y_{1 i}^{*}\right)+2 \cdot \chi \cdot C_{22} \cdot V \cdot J X_{1 i} \cdot \alpha_{1 i} \\
+(-1)^{i+1} \cdot 2 \cdot K y \cdot a \cdot \alpha_{1}^{*}
\end{array}
$$

\subsection{Method of resolution}

The obtained equations form a non-linear system which write $[K(X)]\{X\}=\{B\}$. To resolve the system, we look for a vector, which makes the residue $\{R(X)\}=$ $[K(X)] \cdot\{X\}-\{B\}$ as close as possible to zero. The exact solution is equal to zero. The iterative process converges and stops when $\|n\| \leq \varepsilon$ with $\varepsilon$ a positive constant value $\left(\varepsilon=10^{-14}\right)$.

\section{Simulation results}

We maintain the values below constant, except in case where there is a variation of the constants $\left(R c, K x, \gamma_{e}\right)$

Figures 6 and 7 show the transverse displacement $y_{11}^{*}$, $y_{12}^{*}$ as function of $R c$. For the large curves the leading lateral displacement decreases and it becomes small and constant, and it increases for tight curves. $y_{11}^{*}$ is always positive, $y_{12}^{*}$ is negative. The leading wheelset moves towards the outside of the curve; the trailing wheelset takes a centred position in the railway.

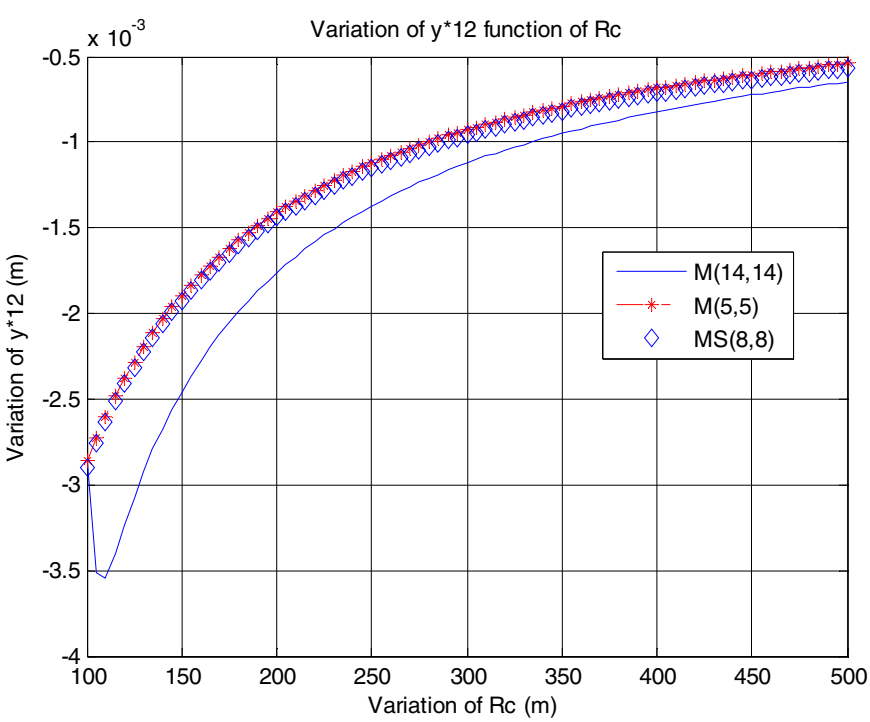

Fig. 7. Trailing wheelset lateral displacement $y *_{12}$ as function of $R c$ (curve radius).

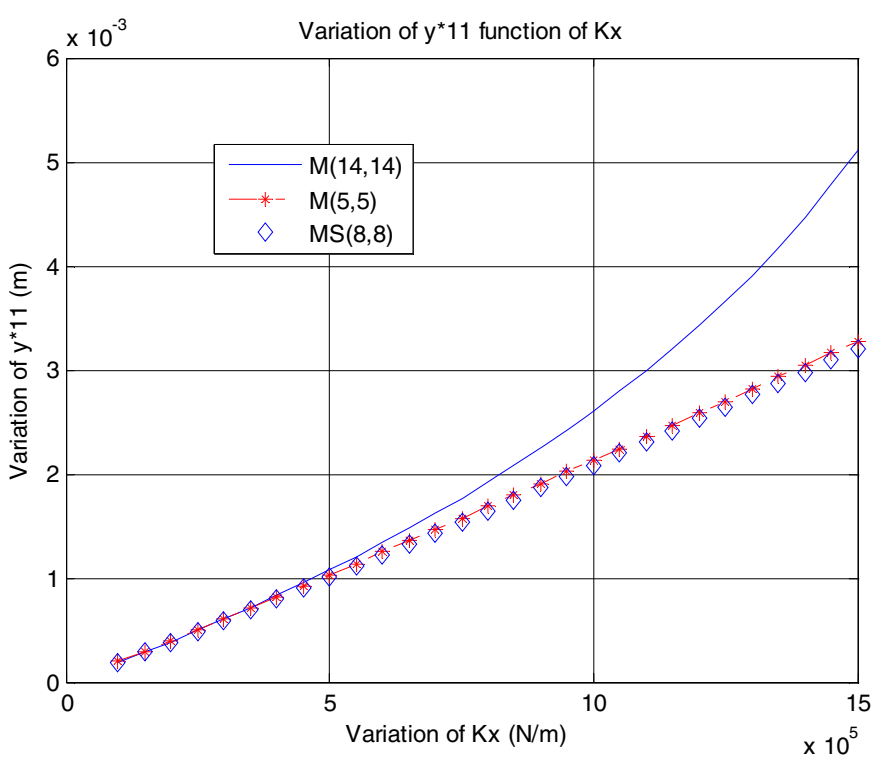

Fig. 8. Wheelset transverse displacement $y_{11}^{*}$ as function of primary suspension $K x$.

Figure 8 shows the variations of the transverse displacement $y_{11}^{*}$, as function of the longitudinal stiffness of primary suspension $K x$. For a constant radius curve $R c$, the transverse displacement increases for high values of longitudinal stiffness of primary suspension $K x$. It shows that stiffness of primary suspension $K x$ have a great influence on the transverse displacement of wheelset. A soft stiffness of primary suspension on leading wheelset reduces the transverse displacement in the traffic on a curve. In this case, the wheel rail wear decreases. The railway company confirms these results by experiment $[5,11]$.

Figure 10 shows the transverse displacement $y_{11}^{*}$ as a function of the wheel conicity $\gamma_{e}$. For a constant radius curve $R c=100 \mathrm{~m}$, the transverse displacement of the leading wheelset decreases when $\gamma_{e}$ increases. For small 
Table 3. Design parameters.

\begin{tabular}{ccccc}
\hline$\gamma_{e}=0.2$ & $\gamma_{0}=0.025$ & $e_{0}=0.75 \mathrm{~m}$ & $\overline{K x}=0{\mathrm{~N} . \mathrm{m}^{-1}}^{-1}$ & $\overline{K y}=3.96 \times 10^{6} \mathrm{~N}^{-1} \mathrm{~m}^{-1}$ \\
$R 1=\infty$ & $d=1 \mathrm{~m}$ & $2 a=1.5 \mathrm{~m}$ & $K x=1,6 \times 10^{6} \mathrm{~N} \cdot \mathrm{m}^{-1}$ & $\delta p=0.07 \mathrm{rd}$ \\
$R^{\prime}=0.3 \mathrm{~m}$ & $r_{0}=0.45 \mathrm{~m}$ & $R c=100 \mathrm{~m}$ & $K y=5 \times 106 \mathrm{~N} \cdot \mathrm{m}^{-1}$ & $m+\widehat{m}=1750 \mathrm{~kg}$ \\
$2 \bar{A}=18.135 \mathrm{~m}$ & $M=3020 \mathrm{~kg}$ & $\bar{d}=1 \mathrm{~m}$ & $\bar{M}=43200 \mathrm{~kg}$ & $R=f\left(\gamma_{0} ; \gamma_{e} ; e_{0} ; r_{0}\right)$ \\
\hline
\end{tabular}

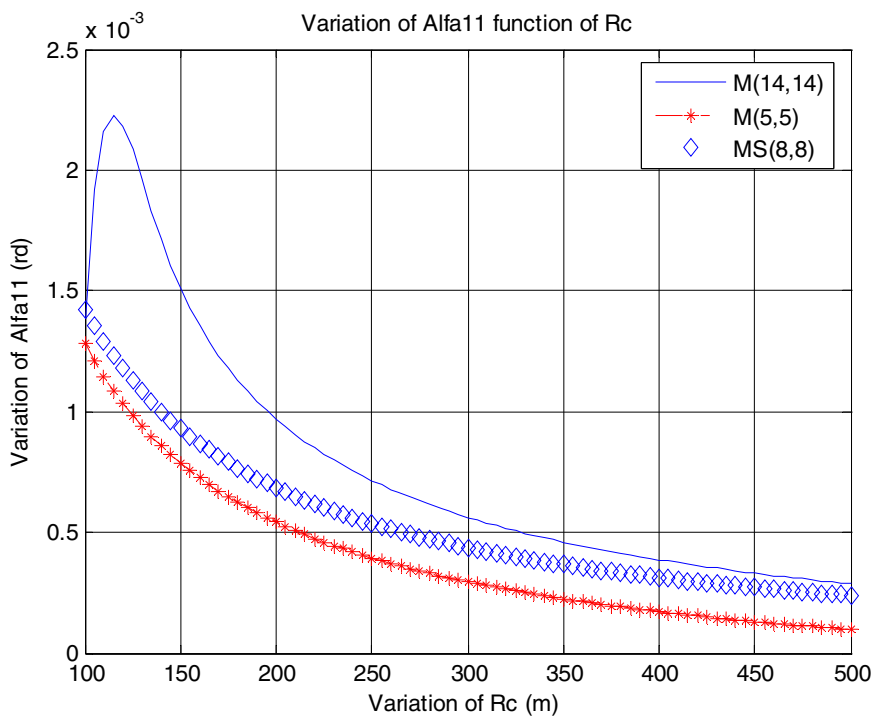

Fig. 9. Leading wheelset yaw angle $\alpha_{11}$ as function of $R c$.

wheel conicity the lateral displacement of leading wheelset increases fastly, there is an evident flange contact between wheel and rail (see Fig. 11), which causes more interaction efforts and yields a high wear of the wheels and rail.

Figure 11 shows that for $\gamma_{e}=0.075$ there is appearance of the effort $F_{11}$ for the models M(14, 14), MS $(8,8)$ and $\mathrm{M}(5,5)$. This effort decreases according to $\gamma_{e}$. It means that we have the advantage of using worn wheels in traffic in curve. The Equation (8) is used to avoid the derailment. For the model $\mathrm{M}(5,5)$ of Figure 11, we obtain:

$$
\begin{array}{r}
F_{11}\left(\gamma_{e}=0.075\right) / N=8.6173 \times 10^{4} / 6.8964 \times 10^{4}= \\
1.2495>1.06 \\
F_{11}\left(\gamma_{e}=0,137\right) / N=7.0507 \times 10^{4} / 6.8964 \times 10^{4} \\
=1.024<1.06
\end{array}
$$

For the equivalent conicity $\gamma_{e}$ between the values $0.075 \rightarrow$ 0.137 the effort between the wheel and the rail cannot be avoided (see Fig. 11), as well as the derailment of the vehicle. For the values between $0.137 \rightarrow 0.25$, the effort between the wheel and the rail cannot avoid, but the derailment of the vehicle is avoided.

Lateral force $F_{11}$ applied by wheelset 1 on the rail as functions of the longitudinal rigidity and the equivalent conicity $\gamma_{e}$ are represented in the Figures 11 and 12 .

Figure 8 gives $y_{11}^{*}=0.0003 \mathrm{~m}$ for all the models for a longitudinal rigidity $K x=0.5 \times 10^{5} \mathrm{~N} . \mathrm{m}^{-1}$. The radius curve is $100 \mathrm{~m}$, Equation (18) gives $y_{0}=0.0169 \mathrm{~m}$. So

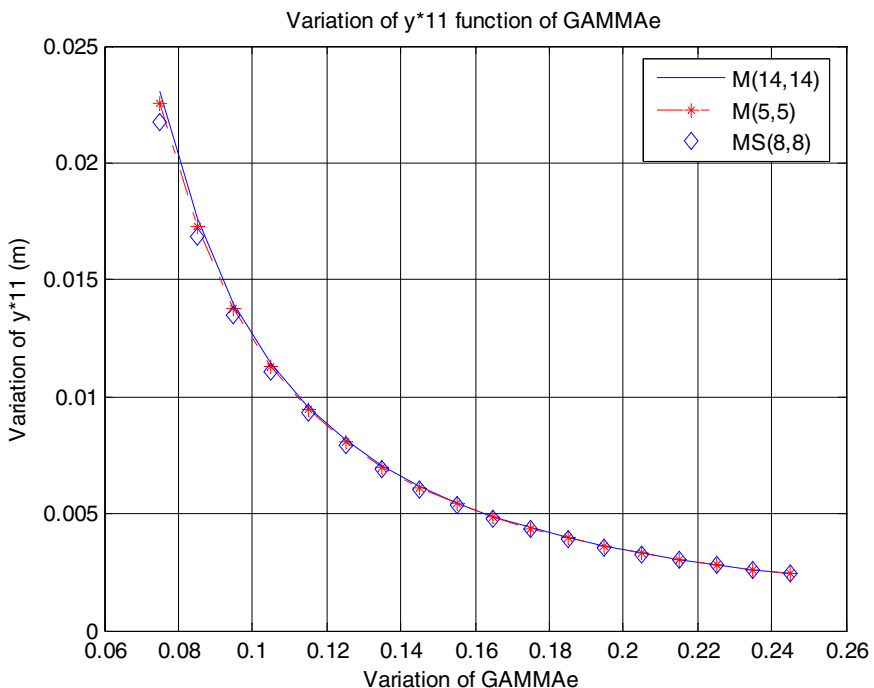

Fig. 10. Trailing wheelset lateral displacement $y_{11}^{*}$ as function of equivalent conicity $\gamma_{e}$.

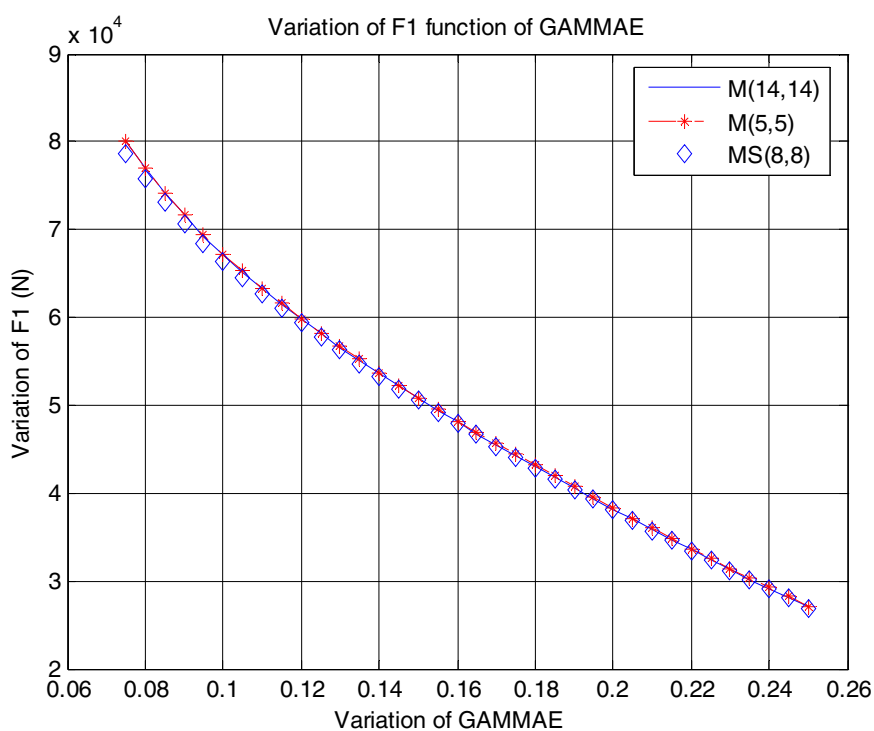

Fig. 11. Lateral force $F_{11}$ applied by wheelset 1 on the rail as function of $\gamma_{e}$.

$K x=0.5 \times 10^{5} \mathrm{~N} . \mathrm{m}^{-1}$, there is appearance of the effort $F 1=2.7 \times 10^{4} \mathrm{~N}$, for the models M $(14,14)$, MS $(8,8)$ and $\mathrm{M}(5,5)$ (see Fig. 12). This effort is proportional to $K x$.

Figure 13 shows the lateral displacement of leading wheelset function of the speed of the vehicle. For curves $R c=150 \mathrm{~m}$, the displacement is more than $0.01 \mathrm{~m}$ and 


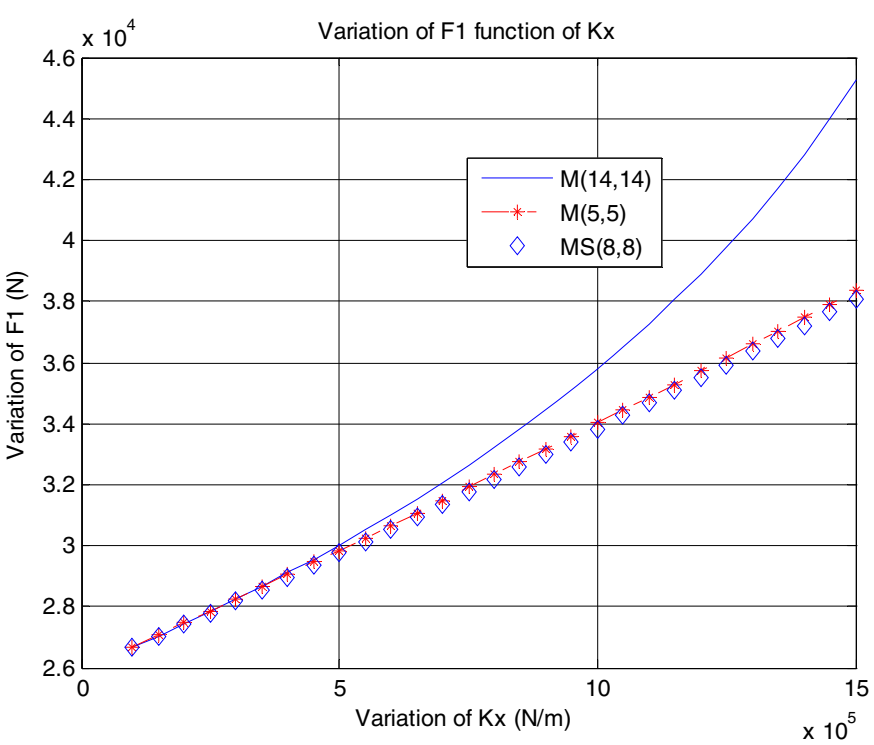

Fig. 12. Lateral force $F_{11}$ applied by wheelset 1 on the rail as function of $K x$.

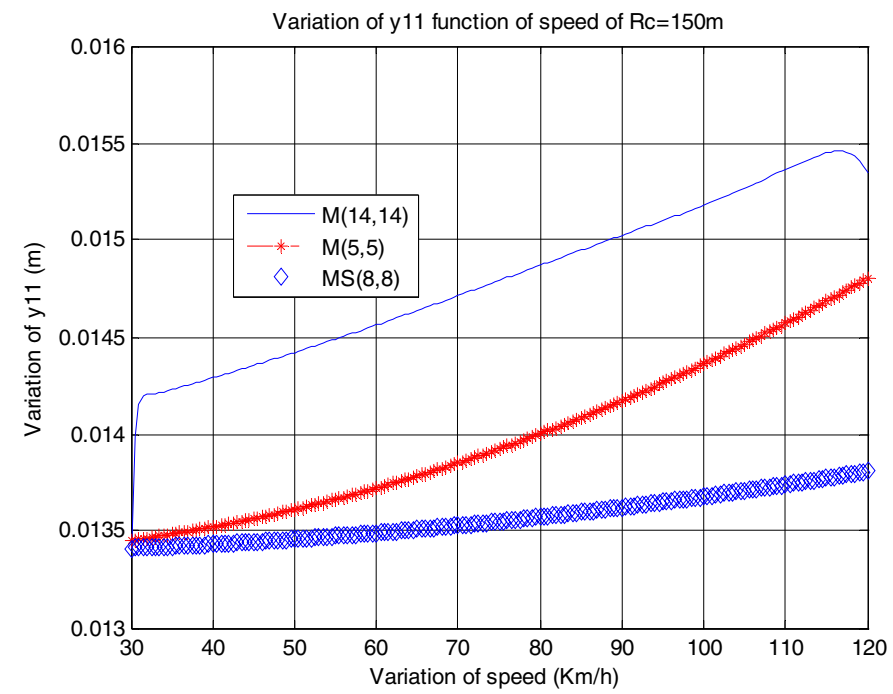

Fig. 13. Trailing wheelset lateral displacement $y_{11}$ as function of the speed of the vehicle.

there is always appearance of the effort $F_{11}$ of the wheel on the rail for the models $\mathrm{M}(14,14)$, $\mathrm{MS}(8,8)$ and $\mathrm{M}(5,5)$.

The model of bogie with two wheelsets represented by the matrix $\mathrm{M}(5,5)$ described perfectly the complete vehicle (see Figs. 6 to 13). This model is simple and does not need much calculation time compared to the model $\mathrm{M}(14,14)$. The model represented by the additional matrix MS $(8,8)$ follows the model M $(5,5)$. The variation of the lateral displacement $y_{11}^{*}, y_{12}^{*}$ and yaw angle $\alpha_{11}$ function of the radius $R c$ curve and the longitudinal rigidity $K x$ shows that the models $\mathrm{M}(14,14), \mathrm{MS}(8,8)$, and $\mathrm{M}(5$, $5)$ stay parallel and follow the same look. All these graphs of the lateral displacements and the yaw angles are very close. Most bibliographical studies [2, 5, 6, 11-13] adapt the model bogie with two wheelsets.

\section{Conclusion}

This study showed that from the fundamental principle of the dynamics of a railway vehicle in relative movement, we obtained the general dynamic equations, which describe the behaviour of the complete vehicle in transit to the curve. From reasonable hypotheses, we obtain the representative quasi-static mathematical model of a railway vehicle in traffic in curve. A bogie and two wheelsets represent this model; every wheelset supports the load of the quarter of the vehicle. The simulation shows that this model describes perfectly the complete vehicle. The advantage of this model is simple and does not need much calculation time compared to a complete vehicle.

\section{Appendix A}

The distances $e_{j}, r_{j}, z_{k i}$ between the centre of inertia of the wheelset and the wheel and rail points of contact are given by:

$$
\begin{gathered}
z_{k i}=\phi \frac{y_{k i}^{2}}{2}-\varepsilon_{0} \gamma_{0} \frac{\alpha_{k i}^{2}}{2} \\
e_{i j}=e_{0}+(-1)^{j} \cdot y_{k i} \gamma_{e} \\
r_{i j}=r_{0}+(-1)^{j} y_{k i} \cdot \gamma_{\mathrm{e}} \\
\gamma_{e}=\frac{R \gamma_{0}}{R-R^{\prime}}\left(\frac{e_{0}+R^{\prime} \gamma_{0}}{e_{0}-r_{0} \cdot \gamma_{0}}\right) \\
\varepsilon_{0}=\left(\left(R+2 r_{0}\right) \gamma_{0}-e_{0}\right) \\
\varphi=\frac{1}{R-R^{\prime}}\left(\frac{e_{0}+R \gamma_{0}}{e_{0}-r_{0} \gamma_{0}}\right)^{2}
\end{gathered}
$$

The expressions of force and moment of centrifugal for the car body, bogie, wheelset; of force and moment inertia Coriolis wheelset's, are given by:

Car body:

$\left\{\begin{array}{l|l}A_{c}^{e} \mid \begin{array}{l}g \\ R \delta p\end{array}\end{array}\right\}=\left\{\begin{array}{l}\bar{M}\left(\frac{V^{2}}{4}\left(\rho_{11}+\rho_{12}+\rho_{21}+\rho_{22}\right)-\overline{h_{0}} \delta_{p}^{\prime \prime}\right) \overrightarrow{y_{\delta p}} \\ \bar{M}\left({\overline{\Omega_{x}}}^{2} \cdot \delta_{p}^{\prime \prime} \vec{X}+{\overline{\Omega_{z}}}^{2} \sigma^{\prime \prime} \vec{Z}\right.\end{array}\right\}_{\bar{G}}$

Bogie:

$$
\begin{aligned}
& \left\{\begin{array}{l}
\left.A_{C_{k}}^{e} \mid \begin{array}{l}
g \\
R \delta p
\end{array}\right\}= \\
\left\{\begin{array}{c}
M_{k}\left(\frac{V^{2}}{4}\left(\rho_{11}+\rho_{12}+\rho_{21}+\rho_{22}\right)\right. \\
\left.-\bar{A}(-1)^{k} \sigma^{\prime \prime}+\left(H+h \bar{h}_{1}-h_{0}\right) \delta_{p}^{\prime \prime}\right) \vec{y}_{\delta p} \\
M_{k}\left({\overline{\Omega_{x k}}}^{2} \cdot \delta_{p}^{\prime \prime} \vec{X}_{k}+{\overline{\Omega_{z k}}}^{2} \sigma^{\prime \prime} \vec{Z}_{k}\right.
\end{array}\right\}_{\bar{G} k}
\end{array}\right.
\end{aligned}
$$




$$
\begin{aligned}
2 T^{R \delta p}\left(C+C_{k}+S_{k i}\right) & =\bar{M}\left(\bar{y}^{\prime 2}+\left({\overline{\Omega_{x}}}^{2}+{\overline{h_{0}}}^{2}\right) \bar{\theta}^{\prime 2}+{\overline{\Omega_{z}}}^{2} \bar{\alpha}^{\prime 2}-2 \cdot \overline{h_{0}} \bar{y}^{\prime} \bar{\theta}^{\prime}\right) \\
& +\sum_{k=1}^{2} M_{k}\left\{\begin{array}{l}
y_{k}^{\prime 2}+\left(\Omega_{x k}^{2}+h_{0}^{2}\right) \theta_{k}^{\prime 2}+\left(H+\overline{h_{1}}-h_{0}\right)^{2} \cdot\left(\delta_{p k}{ }^{\prime}-\delta_{p}{ }^{\prime}\right)^{2} \\
-2 \cdot h_{0} \cdot y_{k}^{\prime} \theta_{k}{ }^{\prime}-2 \cdot h_{0}\left(H+\overline{h_{1}}-h_{0}\right) \cdot\left(\delta_{p k}{ }^{\prime}-\delta_{p}{ }^{\prime}\right) \cdot \theta_{k}{ }^{\prime} \\
+2 y_{k}{ }^{\prime}\left(H+\overline{h_{1}}-h_{0}\right) \cdot\left(\delta_{p k}{ }^{\prime}-\delta_{p}{ }^{\prime}\right)+\Omega_{z k}^{2} \cdot \alpha_{k}{ }^{\prime 2}
\end{array}\right\} \\
& +\sum_{k=1}^{2} \sum_{i=}^{2}\left\{\begin{array}{l}
\Gamma^{2} \cdot y_{k i}^{\prime 2}\left(m \cdot \rho_{x}^{2}+\widehat{m} d^{\prime 2}\right)+m \cdot \rho_{y}^{2}\left(\phi_{k i}^{\prime 2}+2 \cdot \phi_{k i}^{\prime} \cdot \alpha_{k i}^{\prime} \cdot y_{k i} \cdot \Gamma\right) \\
+\alpha_{k i}^{\prime 2}\left(m \cdot \rho_{z}^{2}+\widehat{m} d^{\prime 2}\right)
\end{array}\right\} \\
& +\sum_{k=1}^{2} \sum_{i=}^{2}\left\{\begin{array}{l}
y_{k i}^{\prime 2}+\left(H+\overline{h_{1}}+h_{1}+l\right)^{2} \cdot\left(\delta_{p k}{ }^{\prime}-\delta_{p}{ }^{\prime}\right)^{2}+a^{2} \sigma_{k}^{\prime 2}+ \\
+(m+\widehat{m})\left\{\begin{array}{l}
\left(H+\overline{h_{1}}\right)^{2} \cdot\left(\delta_{p k}{ }^{\prime}-\delta_{p}{ }^{\prime}\right)^{2}-2 y_{k i}^{\prime}\left(H+\overline{h_{1}}\right)\left(\delta_{p k}{ }^{\prime}-\delta_{p}{ }^{\prime}\right)+ \\
2 y_{k i}^{\prime}\left(H+\overline{h_{1}}+h_{1}+l\right)\left(\delta_{p k}{ }^{\prime}-\delta_{p}{ }^{\prime}\right)-2 a(-1)^{-1} \sigma_{k i}^{\prime} \cdot y_{k i}^{\prime}
\end{array}\right\}
\end{array}\right\}
\end{aligned}
$$

Wheelset:

$$
\begin{aligned}
& \left\{\begin{array}{c}
\left.A_{S_{k i}}^{e} \mid \begin{array}{l}
g \\
R \delta p
\end{array}\right\}= \\
\left\{\begin{array}{c}
(m+\widehat{m})\left(\frac{V^{2}}{4}\left(\rho_{11}+\rho_{12}+\rho_{21}+\rho_{22}\right)\right. \\
-\left(\bar{A}(-1)^{k}+a(-1)^{i}\right) \\
\left.\sigma^{\prime \prime}+\left(H+\overline{h_{1}}+h_{1}+l\right) \cdot \delta_{p}^{\prime \prime}\right) \overrightarrow{y_{\delta p}} \\
+\left(m \rho_{x}^{2}+\widehat{m} d^{\prime 2}\right) \delta_{p}^{\prime \prime} \cdot \overrightarrow{X_{k i}} \\
\left(m \rho_{z}^{2}+\widehat{m} \cdot d^{\prime 2}\right) \cdot \sigma^{\prime \prime} \cdot \overrightarrow{Z_{k i}}
\end{array}\right\}_{G_{k i}}
\end{array}\right.
\end{aligned}
$$

Expressions of force and moment inertia Coriolis of the wheelset:

$$
\left\{A_{S_{k i}}^{c} \mid \begin{array}{l}
g \\
R \delta p
\end{array}\right\}=\left\{\begin{array}{l}
\overrightarrow{0} \\
\sigma^{\prime} \varphi_{k i}^{\prime} m\left(\rho_{z}^{2}-\rho_{y}^{2}-\rho_{x}^{2}\right) \cdot \overrightarrow{X_{k i}}+ \\
\delta_{p}^{\prime} \cdot \varphi_{k i}^{\prime} \cdot m\left(\rho_{y}^{2}+\rho_{z}^{2}-\rho_{x}^{2}\right) \cdot \overrightarrow{Z_{k i}}
\end{array}\right\}_{G_{k i}}
$$

The expression of the kinetic energy of the vehicle established the car body, the bogie and four-wheelsets is:

$$
\text { See Equation (A.11) above. }
$$

The expression of elastic power is:

See Equation (A.12) next page.

The power developed by the gravity is:

See Equation (A.13) next page.

\section{Wheel and rail interactive forces}

For traffic of vehicle in a tight curve $(R c<500 \mathrm{~m})$, the reduced sliding of wheels becomes more important. In that case, the force and moment contact are obtained by applying the law of saturation proposed by Johnson and Vermeulen [8], based on the coefficients of Kalker.

$\left(\vec{x}_{\delta p k i} \cdot \vec{u}\left(\gamma_{j}\right)\right)$ defines the tangent plan to the point of contact $I_{k i j} . C_{11}, C_{22}$ and $C_{23}$ are the creep Kalker coefficients, which depend on elasticity modulus, the Poisson's ratio, and the ratio between the semi axes ellipse contact. The creepages in the contact plans (Eq. (A.15)) are derived following the development given by Bettaieb [10].

$$
\left\{\begin{array}{r}
X_{k i j}=C_{11}\left(\vec{\nu}_{k i j} \cdot \vec{x}_{\delta p k i}\right)-\frac{C_{11}^{2}}{3 \mu \cdot N}\left(\vec{\nu}_{k i j} \cdot \vec{x}_{\delta p k i}\right)^{2} \\
\quad+\frac{C_{11}^{3}}{27 \mu \cdot N}\left(\vec{\nu}_{k i j} \cdot \vec{x}_{\delta p k i}\right)^{3} \\
T_{k i j}=C_{22}\left(\vec{\nu}_{k i j} \cdot \vec{u}\left(\gamma_{j}\right)\right)-\frac{C_{22}^{2}}{3 \mu \cdot N} \cdot\left(\vec{\nu}_{k i j} \cdot \vec{u}\left(\gamma_{j}\right)\right)^{2} \\
+\frac{C_{22}^{3}}{27 \mu \cdot N} \cdot\left(\vec{\nu}_{k i j} \cdot \vec{u}\left(\gamma_{j}\right)\right)^{3}
\end{array}\right.
$$

$\vec{v}_{k i j}=\left\{\begin{array}{l}\left((-1)^{j} \frac{e_{0}}{V} \alpha_{k i}{ }^{\prime}-(-1)^{j} \frac{e_{0}}{R c_{k i}}(-1)^{j} \frac{\gamma_{e}}{r_{0}} y_{k i}\right) \vec{x}_{\delta p k i} \\ \left(\frac{\chi}{V} y_{k i}{ }^{\prime}-\alpha_{k i}\right) \vec{u}\left(\gamma_{j}\right)\end{array}\right.$

The longitudinal, lateral creepages in the contact plan are derived following the development $[3,4]$.

$$
\begin{gathered}
X_{k i 1}= \\
C_{11} \cdot \frac{\gamma_{e}}{r_{0}} y_{k i}^{*} \cdot\left\{1-\frac{C_{11}}{3 \mu \cdot N} \frac{\gamma_{e}}{r_{0}}\left|y_{k i}^{*}\right|+\frac{C_{11}^{2}}{27 \mu^{2} \cdot N^{2}}\left(\frac{\gamma_{e}}{r_{0}} y_{k i}^{*}\right)^{2}\right\}
\end{gathered}
$$




$$
\begin{aligned}
& P_{\text {SPRINGS }}=\left\{-\sum_{k=1}^{2} \sum_{j=1}^{2}\left\{\begin{array}{l}
\overline{K_{x k}}\left(-\bar{d}(-1)^{j+1}\left(\overline{\alpha_{k}}-\alpha_{k i}-\sigma_{k}\right)\right)\left(-\bar{d}(-1)^{j+1}\left(\overline{\alpha_{k}^{\prime}}-\alpha_{k i}^{\prime}-\sigma_{k}^{\prime}\right)\right) \\
+\overline{K_{z k}}\left(\bar{d}(-1)^{j+1}\left(\bar{\theta}-\theta_{k}+\delta_{p k}-\delta_{p k i}\right)\right)\left(\bar{d}(-1)^{j+1}\left(\overline{\theta^{\prime}}-\theta_{k}^{\prime}+\delta_{p k}^{\prime}-\delta_{p k i}^{\prime}\right)\right)
\end{array}\right\}\right\}
\end{aligned}
$$

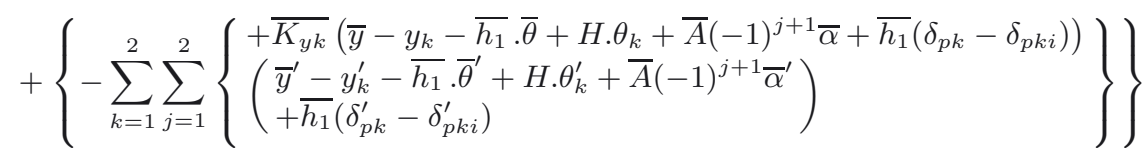

$$
\begin{aligned}
& +\left\{-\sum_{k=1}^{2} \sum_{i=1}^{2} \sum_{j=1}^{2}\left\{\begin{array}{l}
+K_{x k}\left(-d(-1)^{j+1}\left(\alpha_{k}-\alpha_{k i}-\sigma_{k i}\right)\right)\left(\begin{array}{l}
-d(-1)^{j+1} \\
\left(\alpha_{k}^{\prime}-\alpha_{k i}^{\prime}-\sigma_{k i}^{\prime}\right)
\end{array}\right) \\
+K_{y k}\left(\begin{array}{l}
y_{k}-y_{k i}(1-l \Gamma)-h_{1} \theta_{k}+a(-1)^{j+1} \alpha_{k} \\
+h_{1}\left(\delta_{p k}-\delta_{p k i}\right)
\end{array}\right.
\end{array}\right\}\right\} \\
& +\left\{-\sum_{k=1}^{2} \sum_{i=1}^{2} \sum_{j=1}^{2}\left\{\begin{array}{l}
\left(y_{k}^{\prime}-y_{k i}^{\prime}(1-l \Gamma)-h_{1} \theta_{k}^{\prime}+a(-1)^{j+1} \alpha_{k}^{\prime}+h_{1}\left(\delta_{p k}^{\prime}-\delta_{p k i}^{\prime}\right)\right) \\
+K_{z k}\left(d(-1)^{j+1}\left(\theta_{k}-\Gamma y_{k i}+\delta_{p k}-\delta_{p k i}\right)\right) \\
\left(d(-1)^{j+1}\left(\theta_{k}^{\prime}-\Gamma y_{k i}^{\prime}+\delta_{p k}^{\prime}-\delta_{p k i}^{\prime}\right)\right)
\end{array}\right\}\right\}
\end{aligned}
$$

$$
\begin{aligned}
\Pi^{R \delta p}= & \left\{\bar{y}^{\prime}\left(-\bar{M} g \delta_{p}\right)+\overline{\theta^{\prime}}\left(\bar{M} g \overline{h_{0}}\left(\delta_{p}+\bar{\theta}\right)\right)\right\} \\
& \left\{+\sum_{k=1}^{2}\left(\begin{array}{l}
+\theta_{k}^{\prime}\left(M_{k} \cdot g \cdot h_{0}\left(\delta_{p k}+\theta_{k}\right)+y_{k}^{\prime}\left(-M_{k} g \delta_{p k}\right)\right. \\
-M_{k} g\left(\delta_{p k}\left(H+\overline{h_{1}}-h_{0}\right)\left(\delta_{p k}^{\prime}-\delta_{p}^{\prime}\right)-h_{0} \sin \theta\left(\delta_{p k}^{\prime}-\delta_{p}^{\prime}\right)\right)
\end{array}\right)\right\} \\
& \left\{+\sum_{k=1}^{2} \sum_{i=1}^{2} y_{k i}^{\prime}\left(-\left(m_{k i}+\widehat{m}_{k i}\right) \cdot g \cdot \delta_{p k i}-\left(m_{k i}+\widehat{m}_{k i}+\frac{M_{k}}{2}+\frac{\bar{M}}{4}\right) \cdot g \cdot \phi y_{k i} \cdot y_{k i}\right)\right\} \\
& \left.\left\{+\alpha_{k i}^{\prime}\left(m_{k i}+\widehat{m}_{k i}+\frac{M_{k}}{2}+\frac{\bar{M}}{4}\right) \cdot g \cdot \sin \cdot \varphi_{0} \cdot \alpha_{k i}\right)\right\}
\end{aligned}
$$

$$
\begin{array}{r}
T_{k i 1}=C_{22} \cdot \alpha_{k i}\left\{1-\frac{C_{22}}{3 \mu \cdot N}\left|\alpha_{k i}\right|+\frac{C_{22}^{2}}{27 \mu^{2} \cdot N^{2}} \alpha_{k i}^{2}\right\} \\
M_{k i}=-2 . C_{23} \alpha_{k i} ;
\end{array}
$$

We put

$$
\begin{aligned}
& V J X_{k i}=\left\{1-\frac{C_{22}}{3 \cdot \mu \cdot N} \cdot\left|\alpha_{k i}\right|+\frac{C_{22}^{2}}{27 \cdot \mu^{2} \cdot N^{2}} \cdot \alpha_{k i}^{2}\right\} \\
& \operatorname{VJT}_{k i}=\left\{1-\frac{C_{11}}{3 \cdot \mu \cdot N} \frac{\gamma_{e}}{r_{0}} \cdot\left|y_{k i}^{*}\right|+\frac{C_{11}^{2}}{27 \cdot \mu^{2} \cdot N^{2}}\left(\frac{\gamma_{e}}{r_{0}}\right)^{2} \cdot y_{k i}^{* 2}\right\}
\end{aligned}
$$

The dynamic equations of the vehicle in the quasi-static case is: $\mathrm{M}(14,14)(\mathrm{X}) * \mathrm{X}=\mathrm{B}$.

\section{See equation next page.}

See Equation (A.19) next page.

$\mathrm{M}(5,5)$ : Matrix which describes the motions of the two identical bogies and the four wheelsets.

See Equation (A.20) in page 243.

We note by $\operatorname{MS}(8,8)(\mathrm{X})$ the additional matrix.

See Equation (A.21) in page 243. 


$\left[\begin{array}{lllllllllllllll}W . \phi & D_{11} & 0 & 0 & -2 K y & -S & 0 & 0 & 0 & 0 & 0 & 0 & 0 & 0 \\ Q 1 & F & -Q & 0 & 0 & N & 0 & 0 & 0 & 0 & 0 & 0 & 0 & 0 \\ 0 & 0 & W . \phi & D_{12}-2 K y & S & 0 & 0 & 0 & 0 & 0 & 0 & 0 & 0 \\ Q & 0 & Q 2 & F & 0 & N & 0 & 0 & 0 & 0 & 0 & 0 & 0 & 0 \\ 0 & 0 & 0 & 0 & 4 K y & 0 & 0 & 0 & 0 & 0 & 0 & 0 & -2 . \overline{K y} & 0 \\ B 1 & N & -B 1 & N & \frac{P}{2} & A & -\frac{P}{4} & 0 & -\frac{P}{4} & 0 & -\frac{P}{2} & 0 & \frac{P}{2} & -\frac{P}{2} \\ 0 & 0 & 0 & 0 & 0 & 0 & W . \phi & D_{21} & 0 & 0 & -2 K y & -S & 0 & 0 \\ 0 & 0 & 0 & 0 & 0 & 0 & Q 3 & F & -Q & 0 & 0 & N & 0 & 0 \\ 0 & 0 & 0 & 0 & 0 & 0 & 0 & 0 & W . \phi & D_{22} & -2 K y & S & 0 & 0 \\ 0 & 0 & 0 & 0 & 0 . & 0 & Q & 0 & Q 4 & F & 0 & N & 0 & 0 \\ 0 & 0 & 0 & 0 & 0 & 0 & 0 & 0 & 0 & 0 & 4 K y & 0 & 0 & -2 \overline{K y} \\ P / / 4 & 0 & P / 4 & 0 & P / 4 & 0 & B & N & -B & N & -\frac{P}{2} & A & \frac{P}{2} & -\frac{P}{2} \\ 0 & 0 & 0 & 0 & 0 & 0 & 0 & 0 & 0 & 0 & 0 & 0 & 2 \overline{K y} & 2 \overline{K y} \\ B 4 & 0 & B 4 & 0 & -P & M & -B 4 & 0 & -B 3 & 0 & P & M & T & -T\end{array}\right] *\left\{\begin{array}{l}y_{11}^{*} \\ \alpha_{11} \\ y_{12}^{*} \\ \alpha_{12} \\ Y_{1}^{*} \\ \alpha_{1}^{*} \\ y_{21}^{*} \\ \alpha_{21} \\ y_{22}^{*} \\ \alpha_{22} \\ Y_{2}^{*} \\ \alpha_{2}^{*} \\ Y_{1}^{*} \\ Y_{2}^{*}\end{array}\right\}=\left\{\begin{array}{l}b 1 \\ 0 \\ b 3 \\ b 4 \\ b 5 \\ b 6 \\ b 7 \\ b 8 \\ b 9 \\ b 10 \\ b 11 \\ b 12 \\ b 13 \\ 0\end{array}\right\}$

$$
\begin{aligned}
& b 1=b 3=b 7=b 9=(m+\widehat{m}) \gamma_{n c}-W \cdot \phi \cdot y_{0} ; \quad b 13=\bar{M} * \gamma_{n c} ; b 5=b 11=M * \gamma_{n c} ; \\
& b 6=-b 12=2 \overline{K x} \frac{\overline{d^{2}}}{R c} ; \quad b 2=b 8=-b 10=-b 4=2 . K x \cdot \frac{\bar{d} \cdot . a}{R c} ; \\
& A=2 \cdot \overline{K x} \cdot \overline{d^{2}}+4 \cdot K x \cdot d^{2}+4 \cdot K y \cdot a^{2} \cdot ; \quad B=\overline{K x} \cdot \frac{\overline{d^{2}}}{\bar{A}}\left(1+\frac{\bar{A}}{a}\right)+2 \cdot K x \cdot \frac{d^{2}}{a} ; \\
& B 1=\overline{K x} \cdot \frac{\overline{d^{2}}}{a}\left(\frac{2 \bar{A}-a}{2 \bar{A}}\right)+2 K x \cdot \frac{d^{2}}{a} ; B 3=\overline{K x} \cdot \frac{\overline{d^{2}}}{\bar{A}}\left(1-\frac{\bar{A}}{a}\right) ; \quad B 4=\overline{K x} \cdot \frac{\overline{d^{2}}}{\bar{A}}\left(1+\frac{\bar{A}}{a}\right) ; \\
& D_{11}=-2 . C_{22} \cdot \chi V J T_{11} ; \quad D_{12}=-2 \cdot C_{22} \cdot \chi \cdot V J T_{12} ; \\
& D_{21}=-2 \cdot C_{22} \cdot \chi \cdot V J T_{12} ; \quad D_{22}=-2 \cdot C_{22} \cdot \chi \cdot V J T_{22} \\
& F=2 K x \cdot d^{2}+2 \cdot C_{23}-W \cdot \gamma_{0} \cdot \varepsilon_{0} ; \quad H=-\overline{K x} \cdot \overline{\frac{d^{2}}{\bar{A}}}-\overline{K x} \cdot \frac{\overline{d^{2}}}{a} ; \\
& M=-2 \cdot \overline{K x} \cdot \overline{d^{2}} ; \quad N=-2 K x \cdot d^{2} ; \quad P=-2 \cdot \overline{K x} \cdot \overline{\frac{d^{2}}{\bar{A}}} ; \quad Q=-. K x \cdot \frac{d^{2}}{a} ; \\
& Q 1=-. K x \cdot \frac{d^{2}}{a}+2 . C_{11} \cdot \frac{e_{0} \cdot \gamma_{e}}{r_{0}} V J X_{11} ; \quad Q 2=K x \cdot \frac{d^{2}}{a}+2 . C_{11} \cdot \frac{e_{0} \cdot \gamma_{e}}{r_{0}} V J X_{12} ; \\
& Q 3=-. K x \cdot \frac{d^{2}}{a}+2 \cdot C_{11} \cdot \frac{e_{0} \cdot \gamma_{e}}{r_{0}} V J X_{21} ; \quad Q 4=K x \cdot \frac{d^{2}}{a}+2 . C_{11} \cdot \frac{e_{0} \cdot \gamma_{e}}{r_{0}} V J X_{22} ; \\
& S=2 K y \cdot a ; T=2 \cdot \overline{K y} \cdot \bar{A}+2 \overline{K x} \cdot \frac{\overline{d^{2}}}{\bar{A}}
\end{aligned}
$$




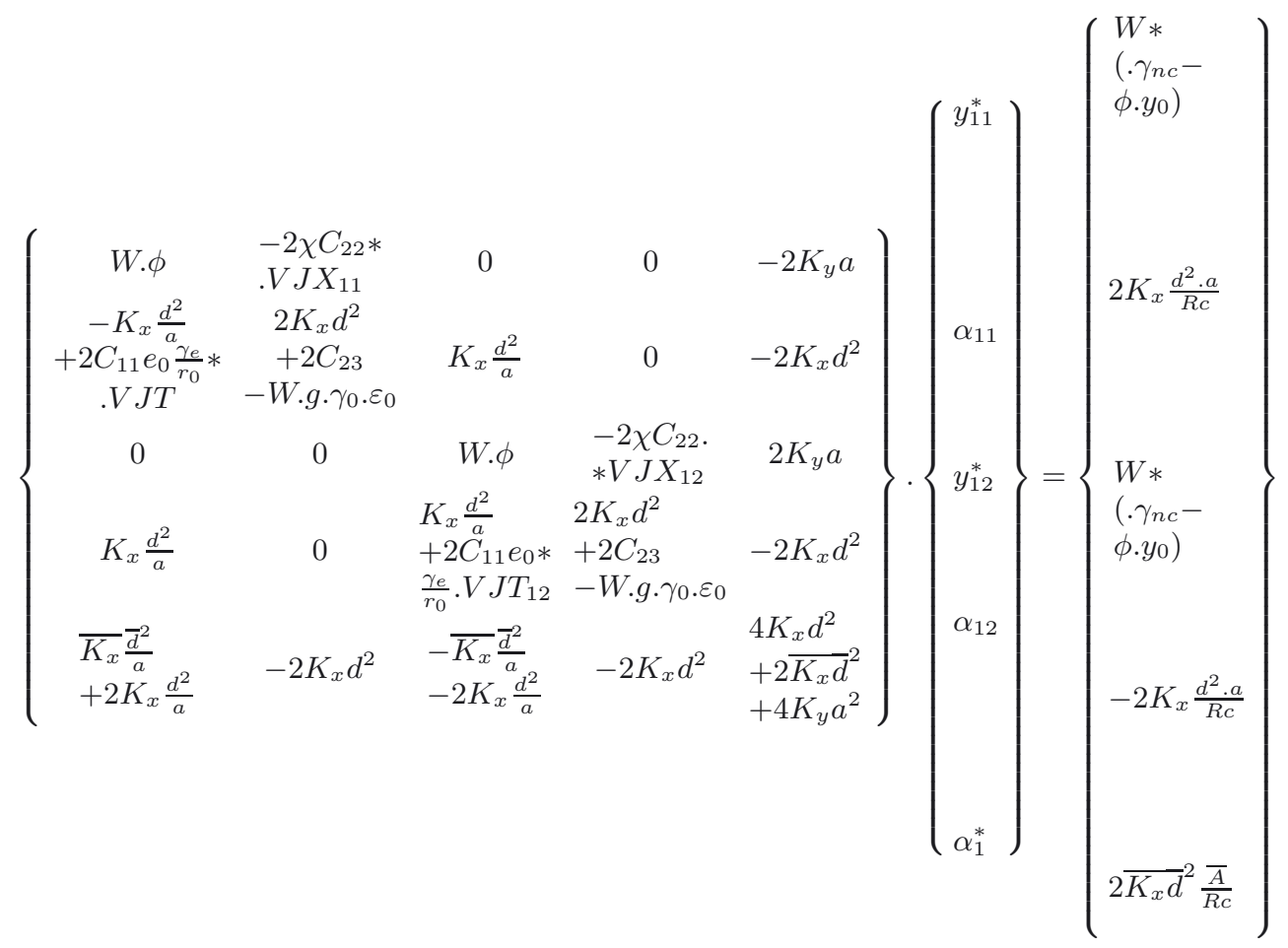

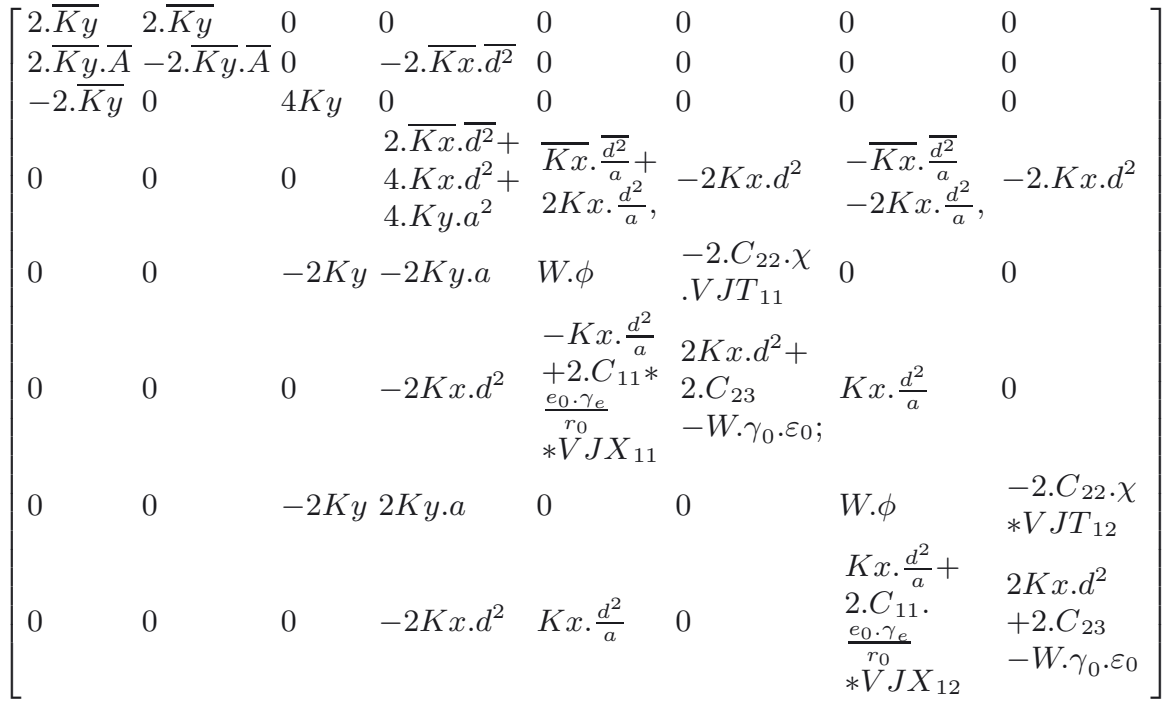

$$
\begin{aligned}
& b s 1=\bar{M}^{*} y_{n c} ; b s 2=0 ; b s 3=\bar{M}^{*} y_{n c} ; b s 4=\overline{2 K x} \frac{\bar{A}}{d^{2}} \frac{\overline{R c}}{R} \\
& b s 6=-b s 8=2 . K x \cdot \frac{d^{2} \cdot a}{R c} ; b s 5=b s 7=(m+\hat{m}) \gamma_{n c}-W \cdot \phi \cdot y_{0} ;
\end{aligned}
$$




\section{References}

[1] H. Bettaieb, Improvement of the railway vehicle performance in circulation in curve, Mécanique \& Industries 9 (2008) 335-346

[2] H. Bettaieb, H. Rejeb, Optimisation des caractéristiques d'un véhicule ferroviaire en stabilité transversale et en circulation en courbe par l'algorithme génétique, Mécanique \& Industries (2008) 347-363

[3] H. Bettaieb, R. Joly, Modélisation d'un véhicule ferroviaire en circulation en courbe, Les Annales Maghrébines de l'ingénieur 7 (1993) 107-120

[4] H. Bettaieb, A. Mlika, Critères de choix des caractéristiques d'un véhicule ferroviaire circulant dans une courbe de faible rayon, Les Annales Maghrébines de l'ingénieur 5 (1991) 195-210

[5] D. Boocok, Steady state motion of railway vehicle on curved track, J. Mech. Eng. 11 (1969) 556-566

[6] Rao V. Dukkipati, S. Narrayana Swamy, Lateral stability and steady state curving performance of unconventional rail trucks, Mechanism and Machine Theory 36 (2001) $507-521$
[7] J. Elkins, H. Wu, Angle of attack and distance based criteria for flange climb derailment, Vehicle system Dynamics Supplement 33 (1999) 293-305

[8] P.J. Vermeulen, K.L. Johnson, Contact of non Spherical body Transmitting Tangential Forces, J. Appl. Mech. 31 (1964) 338-340

[9] J.J. Kalker, On the rolling contact of two elastic bodies in the presence of dry friction, Ph.D. thesis, Delft University of technology, 1967

[10] H. Bettaieb, Étude de la stabilité transversale et le comportement en courbe d'un véhicule ferroviaire, Doctorat $3^{\mathrm{e}}$ cycle mécanique appliquée à la construction, Université de Paris 6, 1983

[11] R. Joly, Circulation d'un véhicule ferroviaire en courbe de faible rayon, J. Rail Int. (1988) 31-42

[12] A.H. Wickens, The dynamic stability of a simplified four wheeled railway vehicle, Int. J. Solids Struct. 1 (1965) 385-406

[13] K. Zboinski, Dynamical investigation of railway vehicle on a curved track, EJMS 17 (1998) 1001-1020 